\title{
Nonlinear dynamics of a non-ideal autoparametric system with MR damper
}

\author{
Danuta Sado \\ Institute of Machine Design Fundamentals, Warsaw University of Technology, 84 Narbutta Street, 02-524 Warsaw, \\ Poland \\ E-mail:dsa@simr.pw.edu.pl
}

\begin{abstract}
The nonlinear response of a three degree of freedom autoparametric system with a double pendulum, including the magnetorheological (MR) damper when the excitation comes from a DC motor which works with limited power supply, has been examined. The non-ideal source of power adds one degree of freedom which makes the system have four degrees of freedom. The influence of damping force in MR damper on the phenomenon of energy transfer has been studied numerically. Near the internal and external resonance region, except periodic vibration also chaotic vibration has been observed. Results show that MR damper can be used to change the dynamic behavior of the autoparametric system.
\end{abstract}

Keywords: Chaos, semiactive damping, autoparametric vibrations, non-ideal system

\section{Introduction}

This work is about nonlinear dynamics of a three degree of freedom system with double pendulum suspended on flexible element with linear spring and semiactive magnetorheological damper (MR) and the excitation comes from a DC motor with limited power supplied (non-ideal system). This system with viscotic damper and harmonic excitation was studied by Sado and Gajos in previous works [6]. In the present paper, the excitation is made by an electric motor with unbalanced mass $m_{0}$. When we use a non-ideal source of power instead of an ideal one we need to add more one degree of freedom to the system. The first detailed study on the non-ideal vibrating systems is reading material by Kononienko [5]. After this publication problem of non-ideal vibrating systems has been investigated by a number of authors. A complete review of different theories on non-ideal vibrating systems were discussed and presented by Balthazar et al. [3]. I announced that the ideal and non-ideal autoparametric system with one pendulum (with viscotic dampers) excited by DC motor with unbalanced mass was studied by Sado and Kot [7, 8], and with two pendulums connected by SMA spring and also with viscotic dampers was presented by Sado et al. [9]. The experimental and numerical analysis of triple physical pendulum externally excited was investigated by Awrejcewicz et al. [2]. The system with pendulum and with magnetorheological (MR) damper subjected to kinematic excitation near the main parametric resonance region was studied by Kęcik and Warmiński [4]. It was show that changing magnetorheological damping dangerous regions can be eliminated or moved away and that MR damper gives control possibilities.

In the presented paper, the influence of the magneto rheological damper when the excitation comes from a DC motor on the dynamic behavior on the auto parametric system, with double pendulum is discussed. The present paper shows that in this type of non-ideal system one mode of vibrations, may excite or damp another mode, and near the resonance regions except multiperiodic and quasiperiodic vibrations, also chaotic motion there may appear. For characterizing an irregular chaotic response in this work, the time histories, the bifurcation diagrams, Poincaré maps and maximal exponents of Lyapunov for different magnetorheological damping parameters are constructed. When a bifurcation diagram is plotted, various phenomena can be observed, e.g. existence a simple or chaotic attractor and various bifurcations. All these effects are verified in the phase space $[1,10]$. Poincaré maps and maximal exponents 


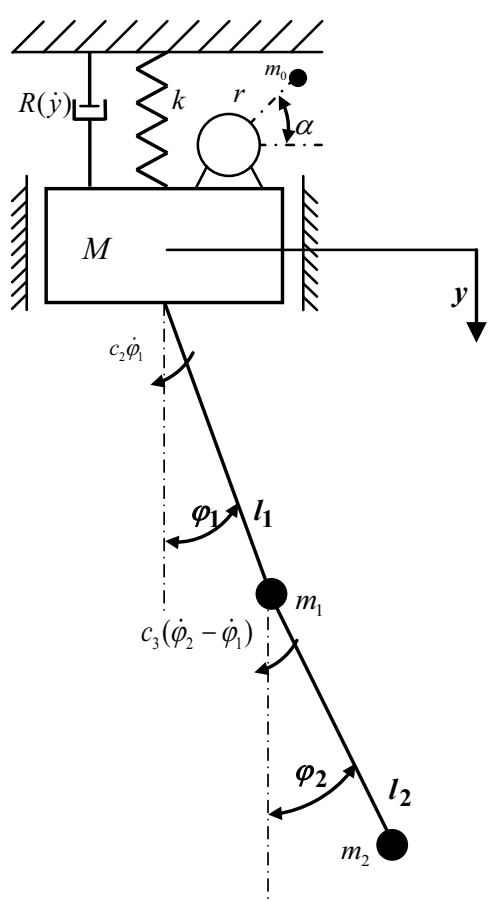

Fig. 1. Schematic diagram of system.

of Lyapunov are available to observe chaos. Results show that magnetorheological (MR) damper can be used to change the dynamic behavior of the autoparametric system.

The presented paper is organized as follows: Section 2 contains a description of the mathematical model of the double pendulum coupled with non-ideal excited oscillator with MR damper, and Section 3 presents exemplary numerical results of this system near internal and external resonance region.

\section{System description and derivation of the governing equations of motion}

The investigated model of an autoparametric system with the double pendulum and a non-ideal source of power are shown in Fig. 1. The system consists of the two coupled pendulums of lengths $l_{1}$ and $l_{2}$ and masses $m_{1}$ and $m_{2}$ hangs down from the body of mass $M$ suspended on a flexible element characterized by a linear elasticity $k$ and magnetorheological (MR) damper of damping force $R(\dot{y})$. It is assumed that the viscous damping forces applied to the hinge opposes motions of the pendulums.

The body of mass $M$ is subjected to an excitation done by an electric motor with an unbalanced mass $m_{o}$. It is assumed that the DC motor is the non-ideal source of limited power. The non-ideal source of power adds one degree of freedom, thus the system has four degrees of freedom. As generalized coordinates are assumed the vertical displacement $y$ of the body of mass $M$ measured from the equilibrium position, the angles $\varphi_{1}$ and $\varphi_{2}$ of deflections of the pendulums measured from the vertical line and angle $\alpha$ which describes the angular displacement of the unbalanced mass $m_{O}$ measured from the horizontal line.

It is assumed that the elasticity force is $S(y)=k\left(y+y_{s t}\right)$, where $y_{s t}$ is the static vertical displacement which can e found from the relation: $\left(M+m_{1}+m_{2}+m_{0}\right) g=k y_{s t}$, where $g$ is the acceleration of gravity. Also is assumed that the damping force acting on the body $M$ is describes by mathematical model which is smooth function of modified Bingham's model suggested by Tang et al. in paper [11] and used by Kęcik and Warmiński in paper [4]. So the damping force is:

$$
R(\dot{y})=C_{1} \dot{y}+C_{4} \tanh (10 \dot{y})
$$


where $C_{1}$ is viscotic damping coefficient and $C_{4}$ is dry friction coefficient.

The kinetic energy $E$ and the potential energy $V$ of the system are:

$$
\begin{aligned}
E= & \frac{1}{2}\left(M+m_{1}+m_{2}+m_{0}\right) \dot{y}^{2}-\left(m_{1}+m_{2}\right) l_{1} \dot{y} \dot{\varphi}_{1} \sin \varphi_{1}-m_{2} l_{2} \dot{y} \dot{\varphi}_{2} \sin \varphi_{2} \\
& +m_{2} l_{1} l_{2} \dot{\varphi}_{1} \dot{\varphi}_{2} \cos \left(\varphi_{2}-\varphi_{1}\right)+\frac{1}{2}\left(m_{1}+m_{2}\right) l_{1}^{2} \dot{\varphi}_{1}^{2}+\frac{1}{2} m_{2} l_{2}^{2} \dot{\varphi}_{2}^{2}+\frac{1}{2}\left(I+m_{0} r\right) \dot{\alpha}^{2}-m_{0} r \dot{y} \dot{\alpha} \cos \alpha \\
V= & -\left(M+m_{1}+m_{2}+m_{0}\right) g\left(y+y_{s t}\right)+m_{2} g l_{2}\left(1-\cos \varphi_{2}\right)+\left(m_{1}+m_{2}\right) g\left(l_{1}-l_{1} \cos \varphi_{1}\right) \\
& +m_{0} g r \sin \alpha+\frac{k\left(y+y_{s t}\right)^{2}}{2}
\end{aligned}
$$

The equation of motions of the system derived as Lagrange's equations take the following form:

$$
\begin{aligned}
& \left(M+m_{1}+m_{2}+m_{0}\right) \ddot{y}-\left(m_{1}+m_{2}\right) l_{1} \ddot{\varphi}_{1} \sin \varphi_{1}-\left(m_{1}+m_{2}\right) l_{1} \dot{\varphi}_{1}^{2} \cos \varphi_{1}-m_{2} l_{2} \ddot{\varphi}_{2} \sin \varphi_{2} \\
& -m_{2} l_{2} \dot{\varphi}_{2}^{2} \cos \varphi_{2}-m_{0} r \ddot{\alpha} \cos \alpha+m_{0} r \dot{\alpha}^{2} \sin \alpha=-C_{1} \dot{y}-C_{4} \tanh (10 \dot{y}) \\
& -\left(m_{1}+m_{2}\right) l_{1} \ddot{y} \sin \varphi_{1}+\left(m_{1}+m_{2}\right) l_{1}^{2} \ddot{\varphi}_{1}+m_{2} l_{1} l_{2} \ddot{\varphi}_{2} \cos \left(\varphi_{2}-\varphi_{1}\right)-m_{2} l_{1} l_{2} \dot{\varphi}_{2}^{2} \sin \left(\varphi_{2}-\varphi_{1}\right) \\
& +\left(m_{1}+m_{2}\right) g l_{1} \sin \varphi_{1}=-C_{2} \dot{\varphi}_{1}+C_{3}\left(\dot{\varphi}_{2}-\dot{\varphi}_{1}\right) \\
& -m_{2} l_{2} \ddot{y} \sin \varphi_{2}+m_{1} l_{1} l_{2} \ddot{\varphi}_{1} \cos \left(\varphi_{2}-\varphi_{1}\right)+m_{2} l_{2}^{2} \ddot{\varphi}_{2}+m_{2} l_{1} l_{2} \dot{\varphi}_{1}^{2} \sin \left(\varphi_{2}-\varphi_{1}\right)+m_{2} g l_{2} \sin \varphi_{2} \\
& =-C_{3}\left(\dot{\varphi}_{2}-\dot{\varphi}_{1}\right)-m_{0} r \ddot{y} \cos \alpha+\left(I+m_{0} r\right) \ddot{\alpha}+m_{0} r g \cos \alpha=L(\dot{\alpha})-H(\dot{\alpha})
\end{aligned}
$$

where $L(\dot{\alpha})$ is a driving torque of DC motor and $H(\dot{\alpha})$ is a resistance torque.

The following dimensionless time and dimensionless parameters have been introduced:

$$
\begin{aligned}
& \tau=\omega_{1} t ; \quad y_{1}=\frac{y}{l_{1}} ; \quad a_{1}=\frac{m_{1}}{M+m_{1}+m_{2}+m_{0}} ; \quad a_{2}=\frac{m_{2}}{M+m_{1}+m_{2}+m_{0}} \\
& a_{3}=\frac{m_{0} r}{\left(M+m_{1}+m_{2}+m_{0}\right) l_{1}} ; \quad \omega_{1}^{2}=\frac{k}{M+m_{1}+m_{2}+m_{0}} ; \quad \omega_{2}^{2}=\frac{g}{l_{1}} ; \quad \omega_{2}^{2}=\frac{g}{l_{1}} ; \quad \beta_{1}=\frac{\omega_{2}}{\omega_{1}} ; c=\frac{l_{2}}{l_{1}} \\
& G_{1}(\dot{\alpha})=\frac{L(\dot{\alpha})-H(\dot{\alpha})}{\left(I+m_{0} r\right) \omega_{1}^{2}} ; \gamma_{1}=\frac{C_{1}}{\left(M+m_{1}+m_{2}+m_{0}\right) \omega_{1}} ; \quad \gamma_{2}=\frac{C_{2}}{\left(M+m_{1}+m_{2}+m_{0}\right) l_{1}^{2} \omega_{1}} \\
& \gamma_{3}=\frac{C_{3}}{\left(M+m_{1}+m_{2}+m_{0}\right) l_{1}^{2} \omega_{1}} ; \quad \gamma_{4}=\frac{C_{4}}{\left(M+m_{1}+m_{2}+m_{0}\right) \omega_{1}} ; \quad q=\frac{m_{0} r l_{1}}{m_{0} r^{2}+I}
\end{aligned}
$$

The characteristic curves $G_{1}(\dot{\varphi})$ of the energy source of DC motor are assumed to be straight lines: $G_{1}(\dot{\varphi})=$ $u_{1}-u_{2} \dot{\varphi}$, where the parameter $u_{1}$ is related to the voltage, and $u_{2}$ is a constant parameter for each model of the motor considered [5]. The $u_{1}$ is the control parameter of the problem.

After transformations, the equations of motion can be written in dimensionless form:

$$
\begin{aligned}
& \ddot{y}-\left(a_{1}+a_{2}\right) \ddot{\varphi}_{1} \sin \varphi_{1}-a_{2} c \ddot{\varphi}_{2} \sin \varphi_{2}-a_{3} \ddot{\alpha} \cos \alpha=\left(a_{1}+a_{2}\right) \dot{\varphi}_{1}^{2} \cos \varphi_{1}+a_{2} c \dot{\varphi}_{2}^{2} \cos \varphi_{2} \\
& -a_{3} \dot{\alpha}^{2} \sin \alpha-y-\gamma_{1} \dot{y}-\gamma_{4} \tanh (10 \dot{y}) \\
& -\left(a_{1}+a_{2}\right) \ddot{y} \sin \varphi_{1}+\left(a_{1}+a_{2}\right) \ddot{\varphi}_{1}+a_{2} c \ddot{\varphi}_{2} \cos \left(\varphi_{2}-\varphi_{1}\right) \\
& =a_{2} c \dot{\varphi}_{2}^{2} \sin \left(\varphi_{2}-\varphi_{1}\right)-\left(a_{1}+a_{2}\right) \beta_{1}^{2} \sin \varphi_{1}-\gamma_{2} \dot{\varphi}_{1}+\gamma_{3}\left(\dot{\varphi}_{2}-\dot{\varphi}_{1}\right) \\
& -\ddot{y} \sin \varphi_{2}+\ddot{\varphi}_{1} \cos \left(\varphi_{2}-\varphi_{1}\right)+c \ddot{\varphi}_{2}=-\dot{\varphi}_{1}^{2} \sin \left(\varphi_{2}-\varphi_{1}\right)-\beta_{1}^{2} \sin \varphi_{2}-\gamma_{3}\left(\dot{\varphi}_{2}-\dot{\varphi}_{1}\right) \\
& -q \ddot{y} \cos \alpha+\ddot{\alpha}=u_{1}-u_{2} \dot{\alpha}-\beta_{1}^{2} q \cos \alpha
\end{aligned}
$$




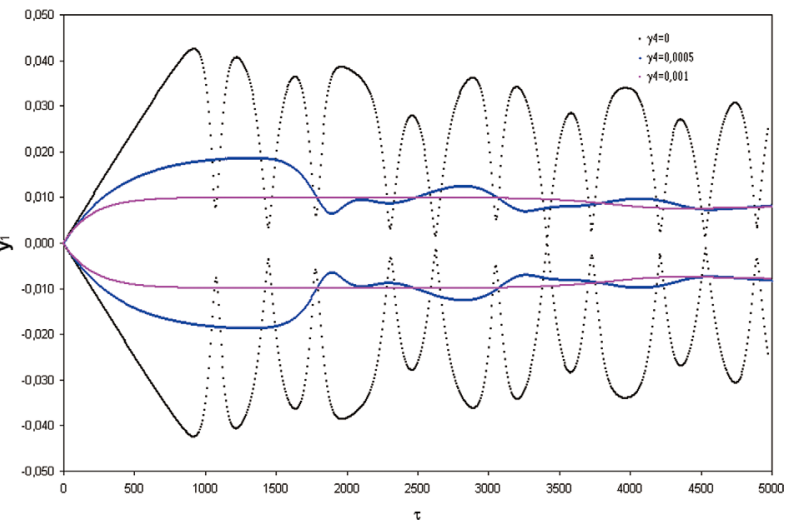

(a)

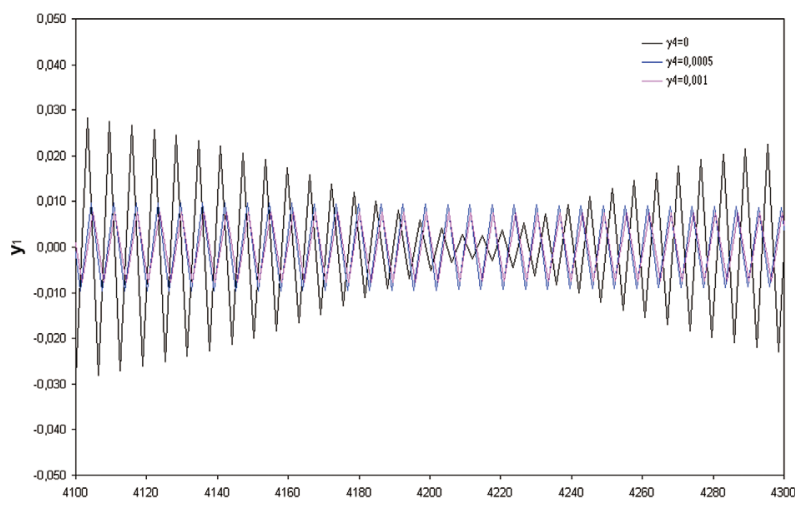

(b)

Fig. 2. Numerical solutions of y for different coefficients $\gamma_{4}$ and for $a_{1}=a_{2}=0.1 ; a_{3}=0.0001 ; \gamma_{1}=0.0001 ; \gamma_{2}=\gamma_{3}=0 ; \beta_{1}=0.65 ; c=$ $1 ; u_{1}=u_{2}=1.5 ; q=0.1$ : (a) envelopes of displacements $y$, (b) time history of $y$ for the region $\tau \in(4100-4300)$.

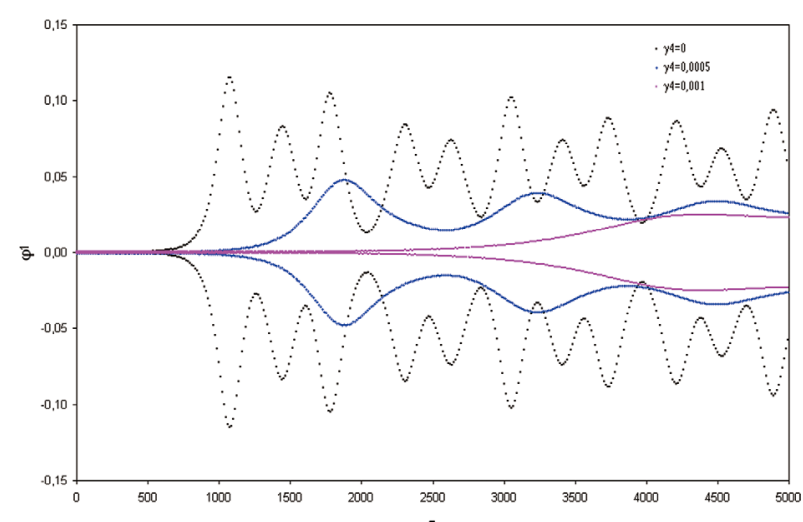

(a)

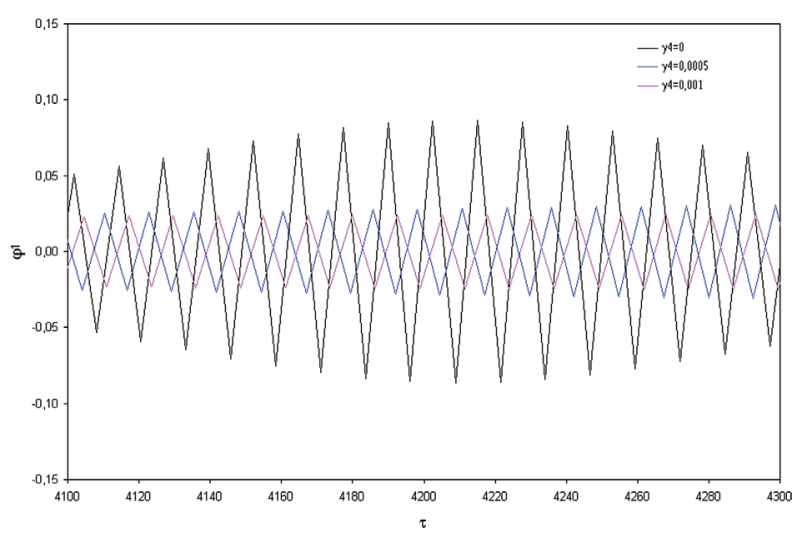

(b)

Fig. 3. Numerical solutions of $\varphi_{1}$ for different coefficients $\gamma_{4}$ and for $a_{1}=a_{2}=0.1 ; a_{3}=0.0001 ; \gamma_{1}=0.0001 ; \gamma_{2}=\gamma_{3}=0 ; \beta_{1}=0.65$; $c=1 ; u_{1}=u_{2}=1.5 ; q=0.1$ : (a) envelopes of displacements $\varphi_{1}$, (b) time history of $\varphi_{1}$ for the region $\tau \in(4100-4300)$.

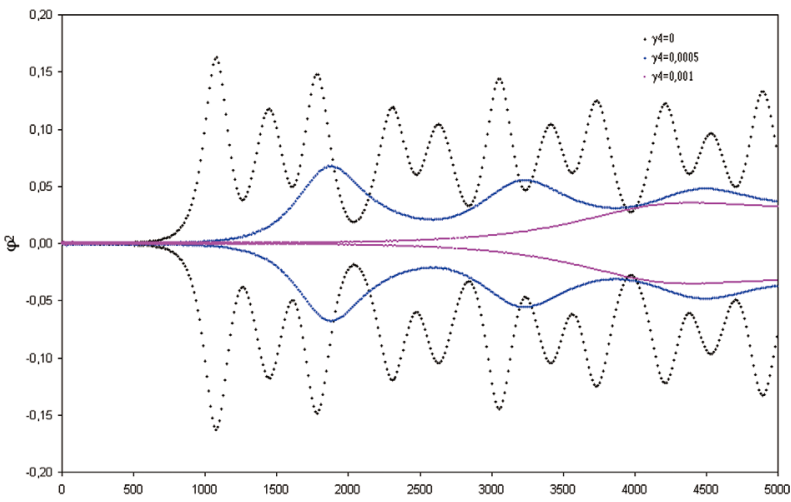

(a)

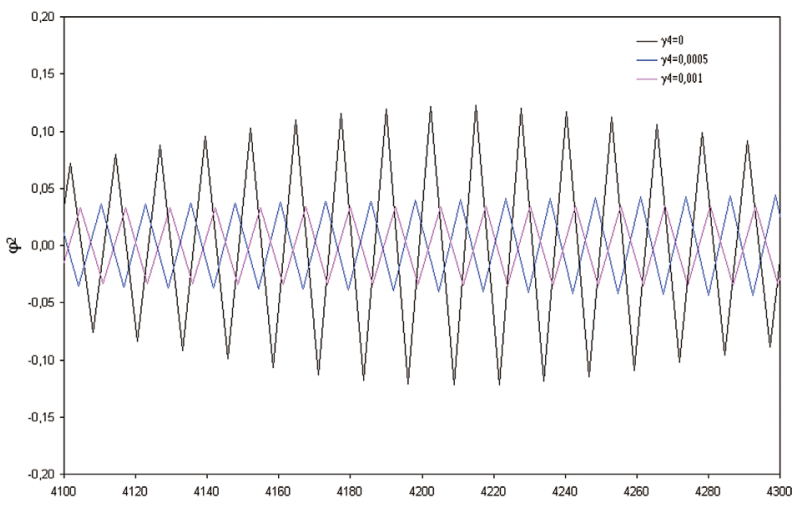

(b)

Fig. 4. Numerical solutions of $\varphi_{2}$ for different coefficients $\gamma_{4}$ and for $a_{1}=a_{2}=0.1 ; a_{3}=0.0001 ; \gamma_{1}=0.0001 ; \gamma_{2}=\gamma_{3}=0 ; \beta_{1}=0.65$; $c=1 ; u_{1}=u_{2}=1.5 ; q=0.1$ : (a) envelopes of displacements $\varphi_{2}$, (b) time history of $\varphi_{2}$ for the region $\tau \in(4100-4300)$. 


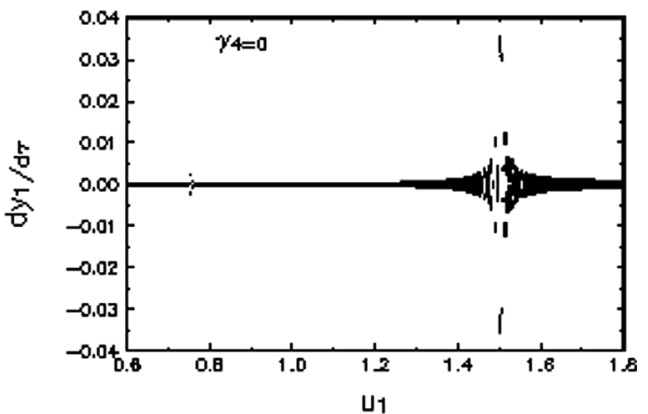

(a)

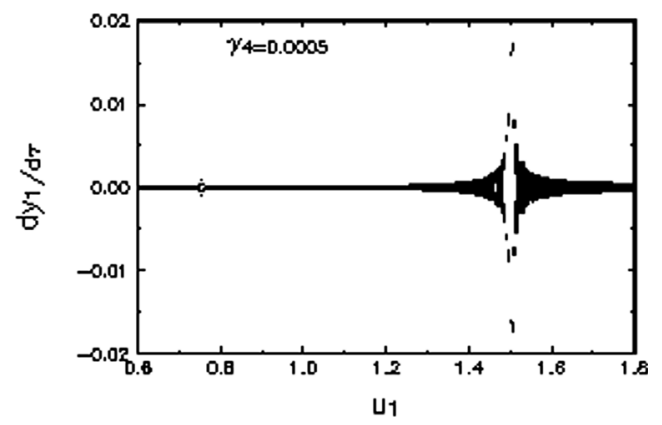

(b)

Fig. 5. Bifurcation diagrams of $y$ for $\gamma_{4}=0$ (a) and $\gamma_{4}=0.0005$ (b) and for: $a_{1}=a_{2}=0.1 ; a_{3}=0.0001 ; \gamma_{1}=0.0001 ; \gamma_{2}=\gamma_{3}=0 ; \beta_{1}=$ $0.65 ; c=1 ; u_{2}=1.5 ; q=0.2$.

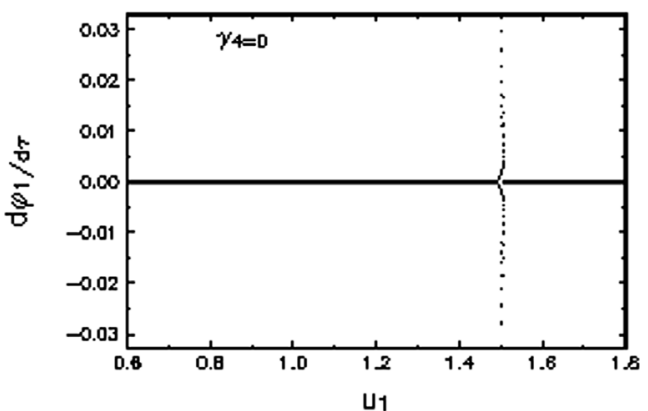

(a)

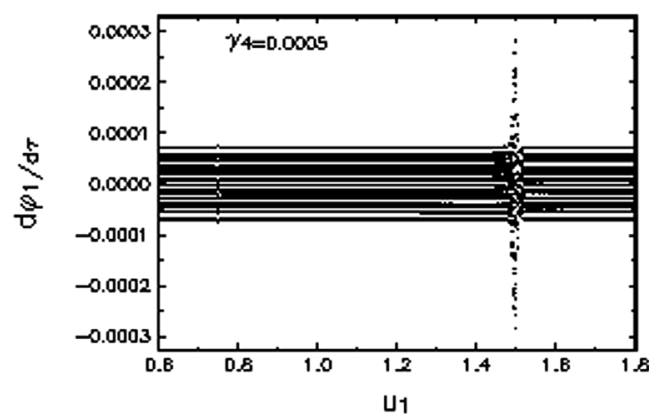

(b)

Fig. 6. Bifurcation diagrams of $\varphi_{1}$ for $\gamma_{4}=0$ (a) and $\gamma_{4}=0.0005$ (b) and for: $a_{1}=a_{2}=0.1 ; a_{3}=0.0001 ; \gamma_{1}=0.0001 ; \gamma_{2}=\gamma_{3}=0$; $\beta_{1}=0.65 ; c=1 ; u_{2}=1.5 ; q=0.2$.

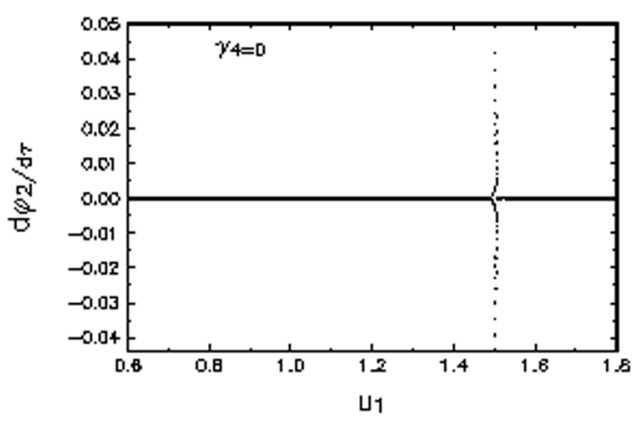

(a)

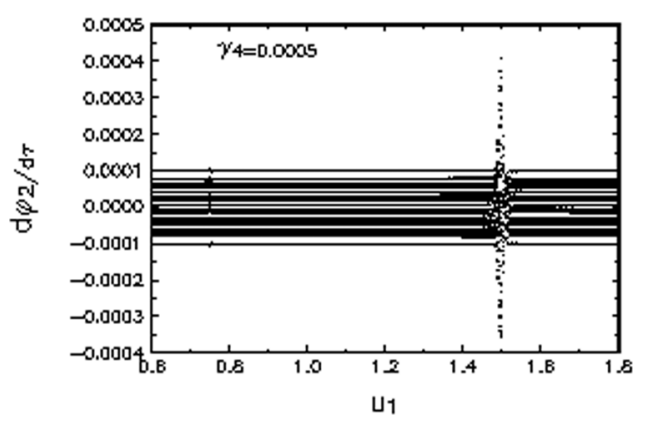

(b)

Fig. 7. Bifurcation diagrams of $\varphi_{2}$ for $\gamma_{4}=0$ (a) and $\gamma_{4}=0.0005$ (b) and for: $a_{1}=a_{2}=0.1 ; a_{3}=0.0001 ; \gamma_{1}=0.0001 ; \gamma_{2}=\gamma_{3}=0$; $\beta_{1}=0.65 ; c=1 ; u_{2}=1.5 ; q=0.2$.

\section{Numerical results}

The governing equations of motion in dimensionless form Eq. (6) have been solved numerically. Calculations have been done for different values of the system parameters and for the following parameters of the engine: $u_{1}=$ $0.2 \div 4, u_{2}=1.5$. The calculations incorporated the following initial conditions: $\dot{\alpha}(0)=1, \phi_{1}(0)=0.005^{\circ}$. Exemplary results the energy transfer for displacement $y, \varphi_{1}$ and $\varphi_{2}$ are presented in Figs 2-4 for different values of magnetorheological damping parameters. All diagrams are presented for viscotic damping parameter $\left(\gamma_{1}=0.00001\right)$ and for different dry friction parameters $\left(\gamma_{4}=0 ; \gamma_{4}=0.0005\right.$ and $\left.\gamma_{4}=0.001\right)$. In Figs 2(a)-4(a) are demonstrated 


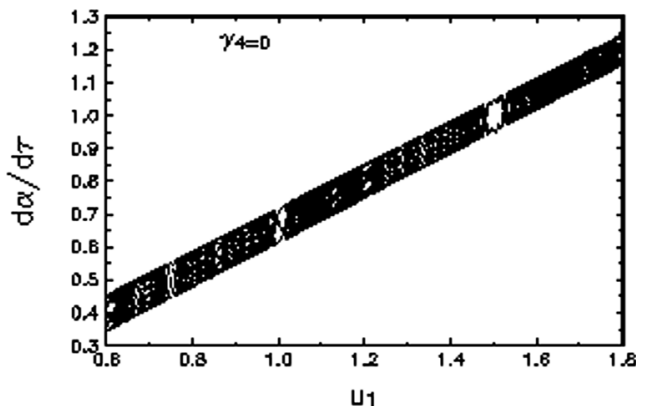

(a)

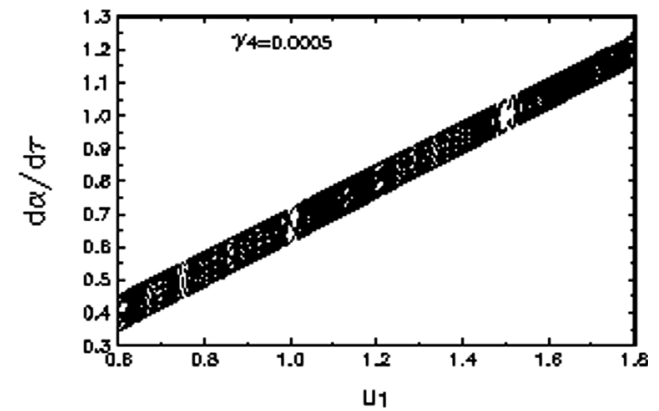

(b)

Fig. 8. Bifurcation diagrams of $\alpha$ for $\gamma_{4}=0$ (a) and $\gamma_{4}=0.0005$ (b) and for: $a_{1}=a_{2}=0.1 ; a_{3}=0.0001 ; \gamma_{1}=0.0001 ; \gamma_{2}=\gamma_{3}=0 ; \beta_{1}=$ $0.65 ; c=1 ; u_{2}=1.5 ; q=0.2$.

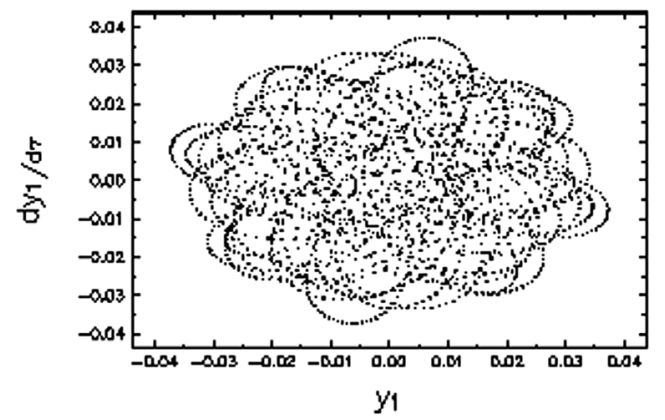

(a)

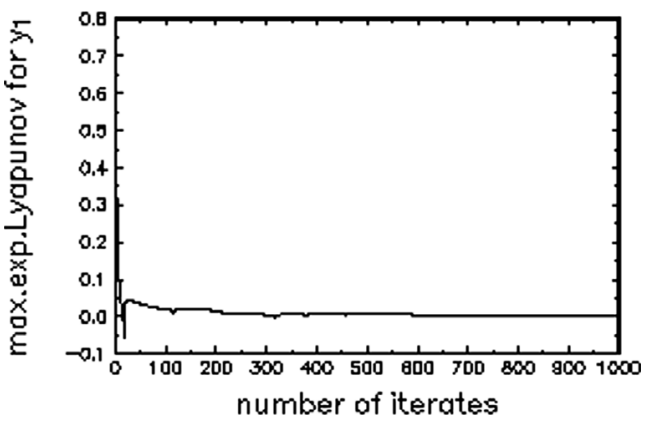

(b)

Fig. 9. Poincaré map (a) and Lyapunov exponents (b) of $y$ for $\gamma_{4}=0$ and for: $a_{1}=a_{2}=0.1 ; a_{3}=0.0001 ; \gamma_{1}=0.0001 ; \gamma_{2}=\gamma_{3}=0 ; \beta_{1}=$ $0.65 ; c=1 ; u_{1}=u_{2}=1.5 ; q=0.2$.

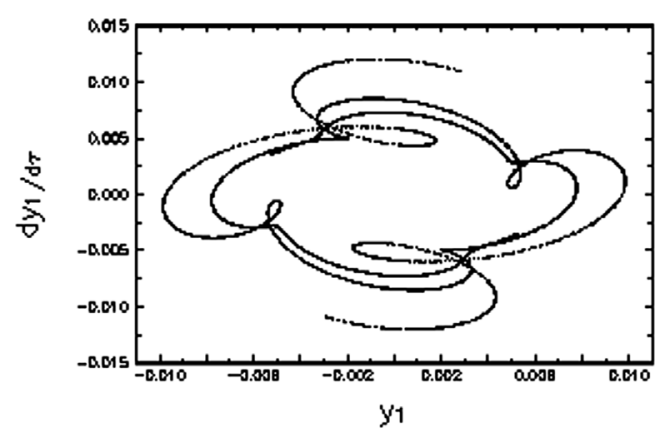

(a)

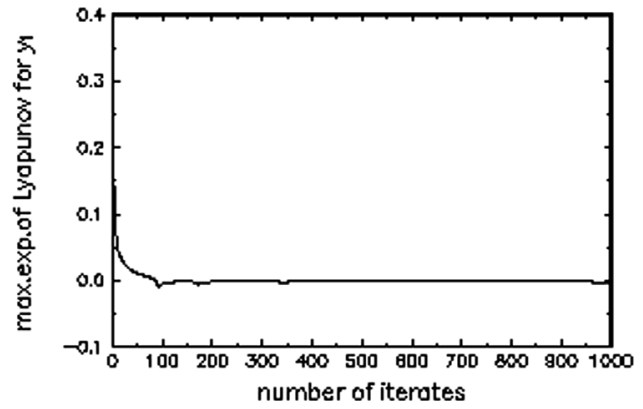

(b)

Fig. 10. Poincaré map (a) and Lyapunov exponents (b) of $y$ for $\gamma_{4}=0.0005$ and for: $a_{1}=a_{2}=0.1 ; a_{3}=0.0001 ; \gamma_{1}=0.0001 ; \gamma_{2}=\gamma_{3}=$ $0 ; \beta_{1}=0.65 ; c=1 ; u_{1}=u_{2}=1.5 ; q=0.2$.

envelopes of displacements $y, \varphi_{1}$ and $\varphi_{2}$ in a long time $\tau \in(0-5000)$ and in Figs 2(b)-4(b) time history of $y, \varphi_{1}$ and $\varphi_{2}$ for the region $\tau \in(4100-4300)$. As can be seen from these Figures for bigger damping parameter $\gamma_{4}$ causes a change of time of the energy transfer cycle. In time $\tau=200$ we can observed 30 periods of vibrations displacement $\mathrm{y}$ and 15 periods motions of pendulums $\varphi_{1}$ and $\varphi_{2}$.

Near the internal and external resonances the system presents some interesting nonlinear phenomena. For characterizing of the system response are constructed [8]: bifurcation diagrams (where the parameter $u_{1}$ related to the voltage is the bifurcation parameter), Poincaré maps and Lyapunov exponents. Bifurcation diagrams versus bifurcation parameter $u_{1}$ for viscotic damping $\left(\gamma_{4}=0\right)$ and for dry friction $\left(\gamma_{4}=0.0005\right)$ are presented: in Fig. 5 for 


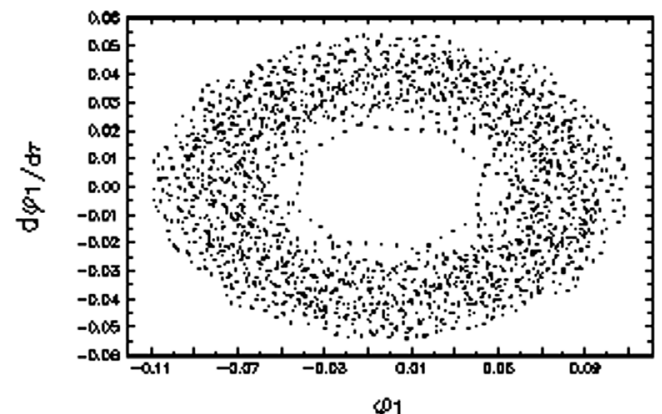

(a)

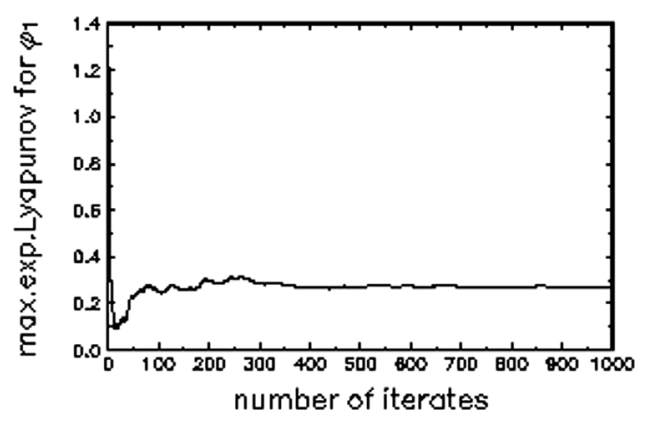

(b)

Fig. 11. Poincaré map (a) and Lyapunov exponents (b) of $\varphi_{1}$ for $\gamma_{4}=0$ and for: $a_{1}=a_{2}=0.1 ; a_{3}=0.0001 ; \gamma_{1}=0.0001 ; \gamma_{2}=\gamma_{3}=0$; $\beta_{1}=0.65 ; c=1 ; u_{1}=u_{2}=1.5 ; q=0.2$.

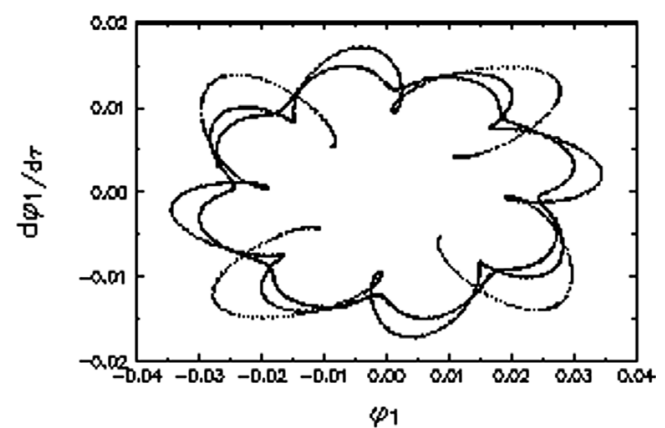

(a)

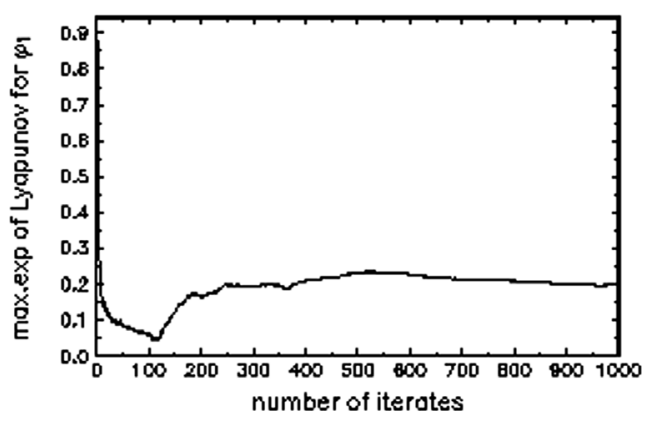

(b)

Fig. 12. Poincaré map (a) and Lyapunov exponents (b) of $\varphi_{1}$ for $\gamma_{4}=0.0005$ and for: $a_{1}=a_{2}=0.1 ; a_{3}=0.0001 ; \gamma_{1}=0.0001 ; \gamma_{2}=\gamma_{3}=$ $0 ; \beta_{1}=0.65 ; c=1 ; u_{1}=u_{2}=1.5 ; q=0.2$.

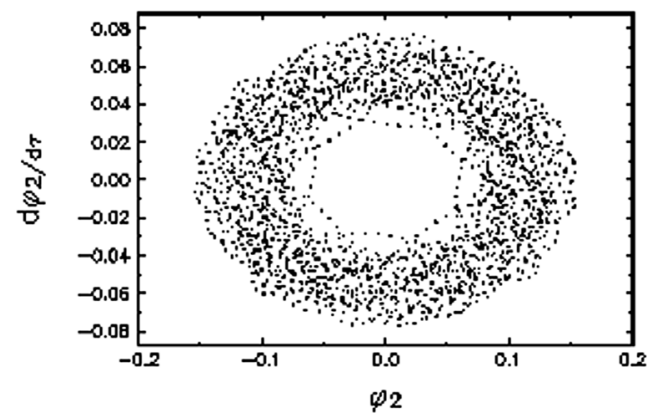

(a)

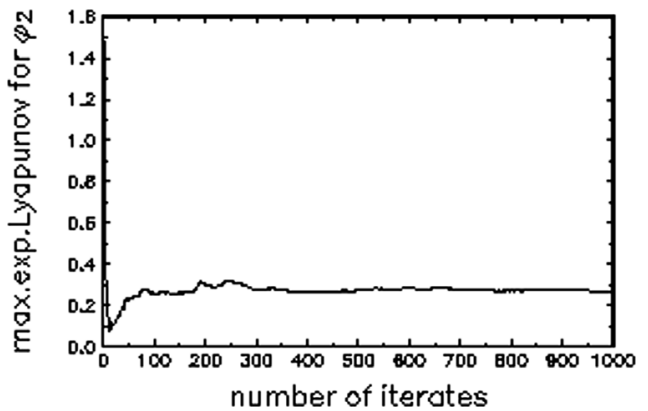

(b)

Fig. 13. Poincaré map (a) and Lyapunov exponents (b) of $\varphi_{2}$ for $\gamma_{4}=0$ and for: $a_{1}=a_{2}=0.1 ; a_{3}=0.0001 ; \gamma_{1}=0.0001 ; \gamma_{2}=\gamma_{3}=0$; $\beta_{1}=0.65 ; c=1 ; u_{1}=u_{2}=1.5 ; q=0.2$.

displacement $y$, in Fig. 6 for displacement $\varphi_{1}$, in Fig. 7 for displacement $\varphi_{2}$, in Fig. 8 for displacement $\alpha$. Poincaré maps and largest Lyapunov exponents for viscotic damping $\left(\gamma_{4}=0\right)$ and for dry friction $\left(\gamma_{4}=0.0005\right)$ are presented in Figs 9-14.

As we see from Fig. 5, the motion of body of mass $M$ may be periodic, quasiperiodic and also chaotic for bifurcation parameter $u_{1}$ near values 1.5. Figures 6 and 7 show that near values $u_{1}=1.5$ we observe chaotic motion of the pendula as well of system with viscotic damping as of dry friction, but values of amplitudes differ. Figure 8 shows influence of the bifurcation parameter $u_{1}$ on the engine velocities.

Exemplary results of the Poincaré maps and maximal exponents of Lyapunov are presented for the bifurcation 


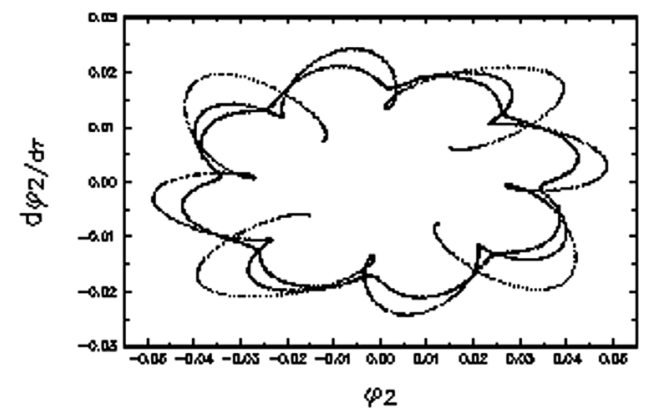

(a)

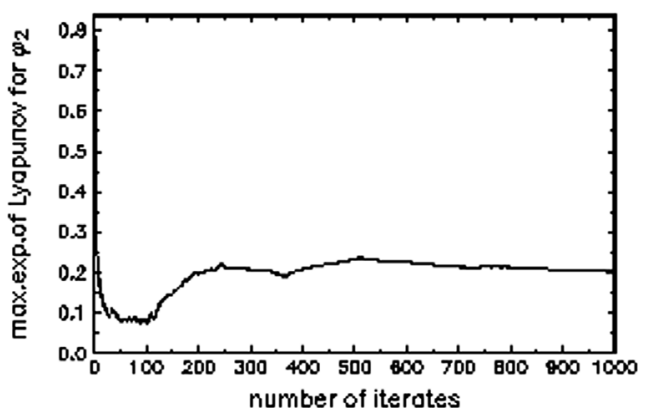

(b)

Fig. 14. Poincaré map (a) and Lyapunov exponents (b) of $\varphi_{2}$ for $\gamma_{4}=0.0005$ and for: $a_{1}=a_{2}=0.1 ; a_{3}=0.0001 ; \gamma_{1}=0.0001 ; \gamma_{2}=\gamma_{3}=$ $0 ; \beta_{1}=0.65 ; c=1 ; u_{1}=u_{2}=1.5 ; q=0.2$.

parameter $u_{1}=1.5$. These diagrams confirm the chaotic nature of motion (Poincaré maps trace the strange attractors for all coordinates and the maximal exponents of Lyapunov corresponding to coordinate y $\varphi_{1}$ and $\varphi_{1}$ are positive).

\section{Conclusion}

This work is concerned with the problem of nonlinear dynamical motion of a non-ideal autoparametric system with double pendulum. Several interesting phenomena have been presented. The behavior of the system near the internal and external resonance frequencies is very important. It has been shown that the examined system exhibits very rich nonlinear dynamics. Except periodic vibrations also chaotic vibrations have been found. The MR damper can be used to change the dynamic behavior of the autoparametric system giving reliable semiactive control possibilities.

\section{Acknowledgments}

The results contained in the paper were presented during the 9 th International Conference on Mathematical Problem in Engineering, Aerospace and Sciences, ICNPAA2012 - Vienna, Austria, July 11-14, 2012.

\section{References}

[1] J. Awrejcewicz, Bifurcation and Chaos in Coupled Oscillators, World Scientific, Singapore, 1991,

[2] J. Awrejcewicz, G. Kudra and G. Wasilewski, Experimental and numerical investigation of chaotic regions in the triple physical pendulum, Special Issue of Nonlinear Dynamics 50(4) (2007), 755-766.

[3] J.M. Balthazar, D.T. Mook, H.I. Weber, H.M.L.R.F. Brasil, A. Fenili, D. Belato and J.L.P. Felix, An overview on non-ideal vibrations Meccanica 38 (2003), 613-621.

[4] K. Kęcik and J. Warmiński, Dynamics of an autoparametric pendulum-like system with a nonlinear semiactive suspension, Hindawi Publishing Corporation, Mathematical Problem of Engineering, doi:10.1155/2011/451047.

[5] V.O. Kononenko, Vibrating Systems with Limited Power Supply, Illife Books, London, 1969.

[6] D. Sado and K. Gajos, Note on chaos in three degree of freedom dynamical system with double pendulum, Meccanica 38 (2003), $719-729$.

[7] D. Sado and M. Kot, Nonlinear oscillations of a coupled autoparametrical system with ideal and nonideal sources of power, Mathematical Problem in Engineering, (2006), 1-20, doi 10.1155/MPE/82691.

[8] D. Sado and M. Kot, Chaotic vibration of an autoparametrical system with a non ideal source of power, Journal of Theoretical and Applied Mechanics 45(1) (2007), 119-131.

[9] D. Sado, M. Pietrzakowski and K. Gajos, Pseudoelastic effect in autoparametric non-ideal system with SMA spring, Dynamical Systems, Theoretical \& Applied Mechanics Letters 2(4) (2012), 04013.

[10] W. Szemplińska-Stupnicka, Chaos, Bifurcations and Fractals Around Us, World Scientific, London, (2003),

[11] D. Tan, H. Gavin and E. Dwell, Study of airfoil gust response alleviation using on electromagnetic dry friction damper Part I: Theory, Journal of Sound and Vibration, 3-5, 269, (2004), 853-874. 

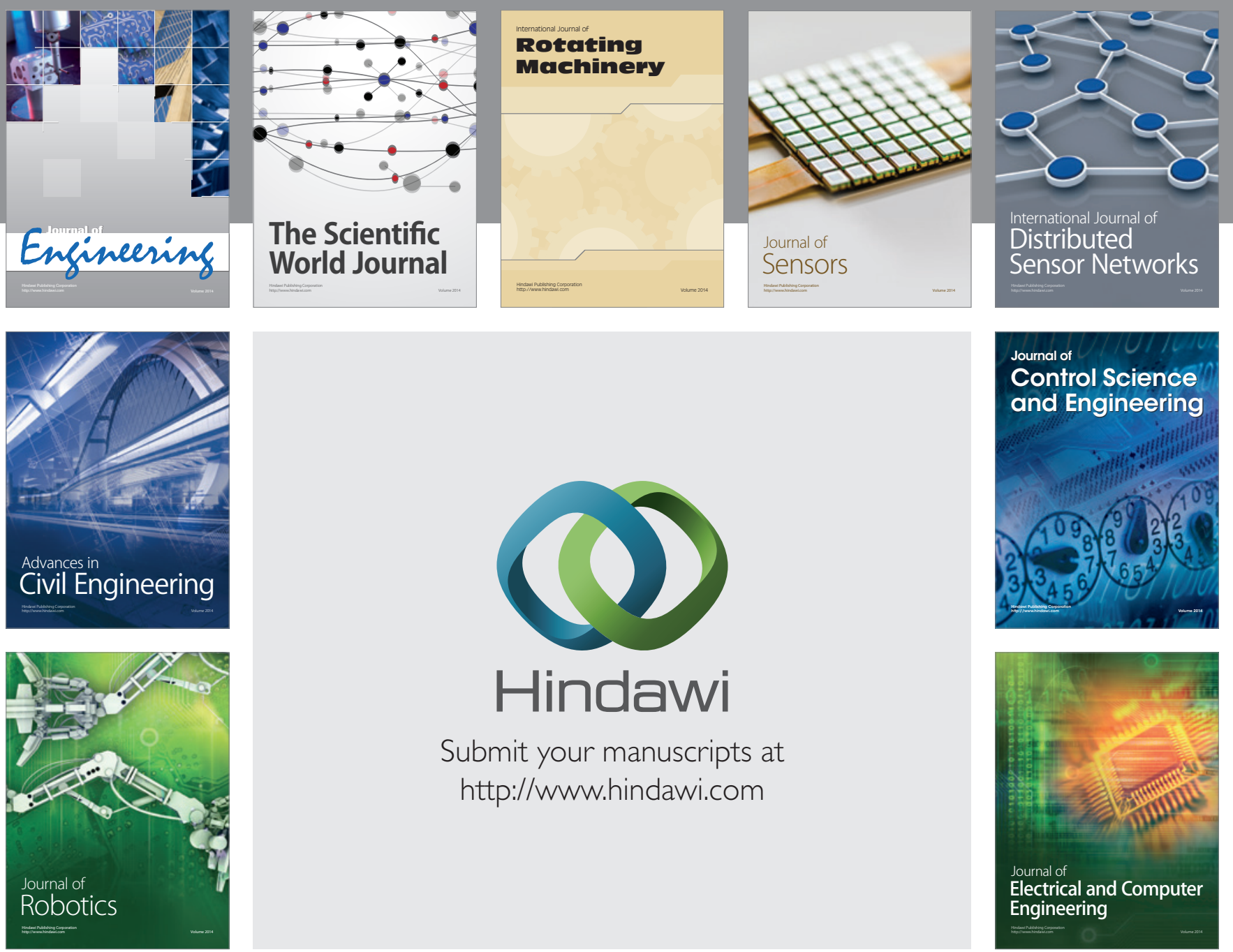

Submit your manuscripts at

http://www.hindawi.com
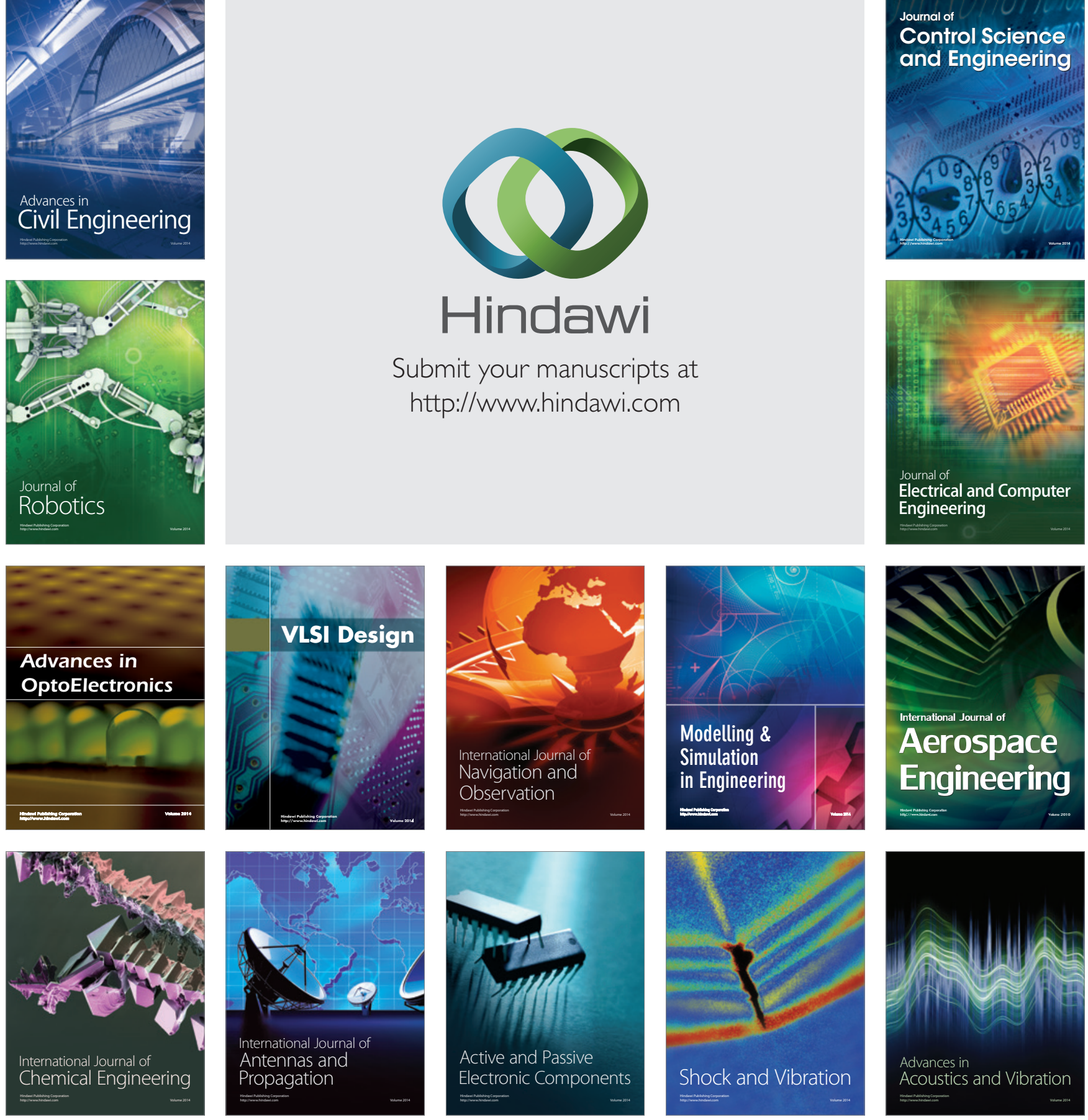